\title{
A Judging Method of Residual Stress Based on Improved HHT and Correlation Analysis
}

\author{
Zhou Xiao-long, a , Liu Wei-na', b, Jiang Zhen-hai ${ }^{2,}$, Ma Feng-lei $^{2}$ \\ ${ }^{1}$ College of Mechanical and Electric Engineering,Changchun University of Science and \\ Technology,Changchun 130022, Jilin, P.R. China \\ ${ }^{2}$ School of Mechatronic Engineering, Changchun University of Technology, Changchun 130012, \\ Jilin, P.R. China \\ az85217479@163.com, bwhliu77@163.com
}

Keywords: HHT; residual stress; EMD; correlation analysis.

Abstract. It is well known that the engineering properties of materials and structural components can be considerably influenced by residual stress. In this paper, a new judging method of residual stress based on improved Hilbert-Huang transform (HHT) is proposed. Two types of high strength aluminum alloy plate with or without the residual stress is analyzed by this technique. The cross correlation coefficients of each intrinsic mode function (IMF) under the two conditions are given. The results show that the changing regulations can be got and the IMF component which is great influenced by the residual stress can also be found out by this method.

\section{Introduction}

It is well known that residual stress occur in many materials and structures, and residual stress are becoming more and more important in the precision engineering fields which considerably influence the engineering properties [1-2]. Accordingly, study of residual stress is very important for mechanical components designing and manufacturing [3-4].

In this paper, a novel judging method of residual stress based on improved HHT is proposed. This paper starts with a brief introduction of HHT and correlation analysis method, and then a detail description of the experimental study is introduced. And the experimental results are discussed in detail. Finally the paper is concluded a summary of the main findings based on the obtained result.

\section{Improved Hilbert-Huang Transform}

EMD technique is the core content of HHT. However, this technique has some problems, such as the presence of oscillations of very disparate amplitude in a mode, or the presence of very similar oscillations in different modes, named as "mode mixing" [5]. In order to solve this problem, Torres proposed an complete ensemble empirical mode decomposition with adaptive noise (CEEMDAN) method. CEEMDAN algorithm can be described as follows:

Let $E_{k}(\cdot)$ be the operator which produces the $k$ th mode obtained by EMD based on BLEME technique; let $w^{(i)}$ be white noise with different amplitudes; and defined the operator $\langle\cdot\rangle$ which calculates the average value of the signal. For the targeted signal $x^{(i)}=x+\beta_{0} w^{(i)}\left(\beta_{0}>0\right)$ the CEEMDAN algorithm can be described as follows:

(1) Decompose $x^{(i)}(i=1,2, \ldots, I)$ by EMD based on EMD technique to obtain their first mode and compute

(2) Calculate the first residual of $x^{(i)}$

$$
\tilde{d}_{1}=<E_{1}\left(x^{(i)}\right)>\quad(k=1)
$$

$$
r_{1}=x-\tilde{d}_{1}
$$


(3) Decompose $r_{1}+\beta_{1} E_{1}\left(w^{(i)}\right)(i=1,2, \ldots, I)$ until obtain the first mode and define the second CEEMDAN IMF component as:

$$
\tilde{d}_{2}=<E_{1}\left(r_{1}+\beta_{1} E_{1}\left(w^{(i)}\right)>\right.
$$

(4) For $k=2, \ldots, K$ calculate the $k$ th residual:

$$
r_{k}=r_{(k-1)}-\tilde{d}_{k}
$$

(5) Decompose $r_{k}+\beta_{k} E_{k}\left(w^{(i)}\right)(i=1,2, \ldots, I)$ and get their first mode by EMD based on EMD technique and define the $(k+1)$ th OFCEEMDAN mode as:

$$
\tilde{d}_{(k+1)}=<E_{1}\left(r_{k}+\beta_{k} E_{k}\left(w^{(i)}\right)>\right.
$$

(6) Go to step (4) for the next $k$.

When obtained residual is not satisfy IMF conditions or it does not have at least two extreme, decomposition is finished. The final residual can define as

$$
r_{k}=x-\sum_{k=1}^{K} \tilde{d}_{k}
$$

The total number of IMF component is $K$ and the signal $x$ can be expressed as

$$
x=\sum_{k=1}^{K} \tilde{d}_{K}+r_{K}
$$

From Eq.(7) we can know that the decomposition is complete and it produces an exact reconstruction of original signal $x$.

\section{Cross Correlation Function}

Study the relationship between the two variables, called the correlation analysis. In correlation analysis, study the correlation between two signals at any two different moments, the correlation function is always used. It indicates the degree of correlation between the two signals at any two different moments.

For two consecutive signals $x(t)$ and $y(t)$, their correlation function is defined as:

$$
R_{x y}(\tau)=\frac{1}{T} \int_{0}^{T} x(t) y(t+\tau) d t
$$

Where $\tau$ is the delay time, $2 T$ is the sampling point.

In dealing with practical problems, in order to specifically express the degree of correlation between the two variables, the cross correlation coefficient $\rho_{x y}(\tau)$ is always selected. It is defined as:

$$
\rho_{x y}(\tau)=\frac{R_{x y}(\tau)}{\sqrt{R_{x}(0) R_{y}(0)}}
$$

Where $\left|\rho_{x y}(\tau)\right| \leq 1, R_{x}(0)$ and $R_{y}(0)$ representation the value of the cross correlation function of $x(t)$ and $y(t)$ at $\tau=0$. The more $\rho_{x y}(\tau)$ closer to 1 , the greater the degree of similarity between the two variables.

\section{Experimental Studies}

Materials used for the test is 7075 high strength aluminum alloy plates. The size of the sample is $500 \mathrm{~mm} \times 50 \mathrm{~mm} \times 8 \mathrm{~mm}$. One of the samples is surface milling and hardening, another sample without these treatments.

The experimental setup includes testing specimen, oscillation and analysis system. The 
oscillation and analysis system is composed of one acceleration sensor, hammer, Brüel \& Kjær 3050 data collector, PULSE analysis software and a computer. The sampling frequency is $6400 \mathrm{~Hz}$ and the sampling is $5 \mathrm{~s}$. The vibration signals of the specimen under different stress are shown in Fig.1.

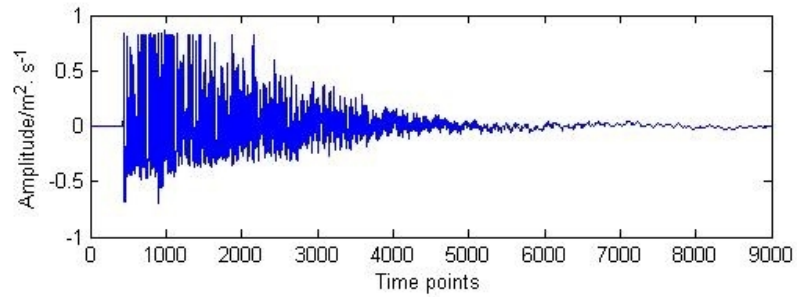

(a) Signal of the specimen without residual stress

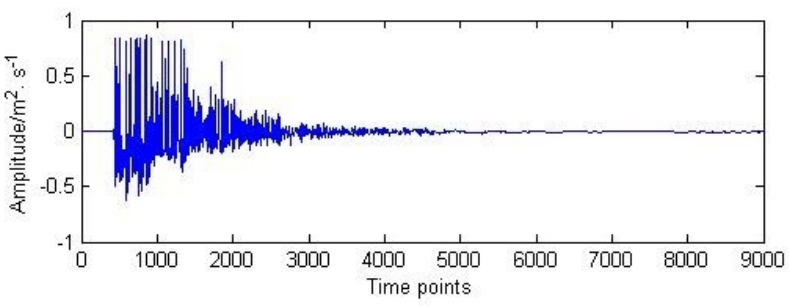

(b) Signal of the specimen with residual stress

Fig.1 Signal of the specimen with different stress.

From Fig.1 we know that even the amplitude of the spacemen have some differences. But more information about the specimen or its state can not get.

The frequency spectrum of the specimen can be seen in Fig.2. All frequency spectral components are rich, there are 4 main frequencies in 2 figures, and in Fig.2 (b) there are more frequencies in low bands than Fig.2 (a). But the frequency spectrum can only reflect the overall composition of the frequency components. It is difficult to find the cause of the above phenomenon and also difficult to get inner principles about them.

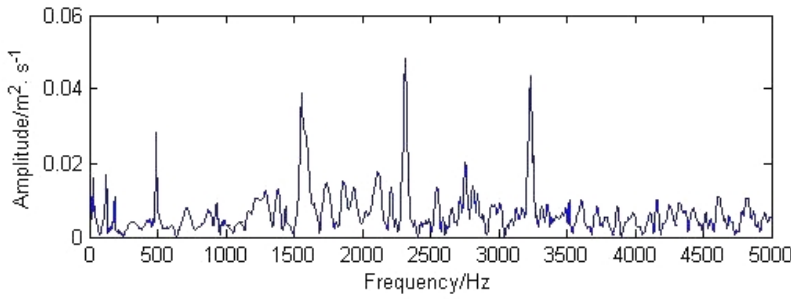

(a) The specimen without residual stress

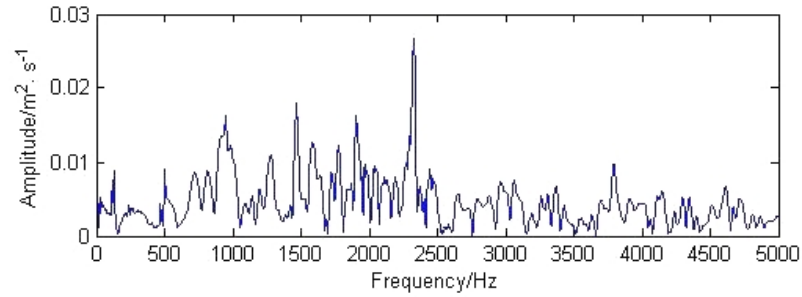

(b)T he specimen with residual stress.

Fig.2 Frequency spectrum of the specimen with different stress.

The signals are decomposed by CEEMDAN method, the ensemble size $M=100$ and noise standard deviation $\varepsilon=0.1$, they all get 8 IMF components, the decomposition result are shown in Fig.3.

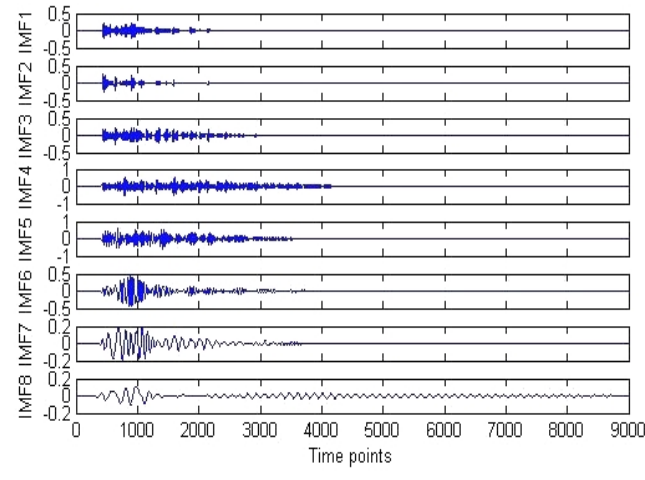

(a)

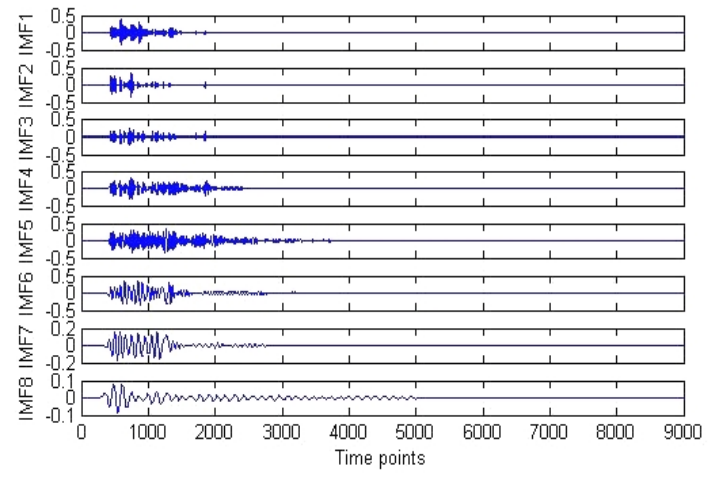

(b)

Fig.2 Frequency spectrum of the specimen with different stress. (a) Frequency spectrum of the specimen without residual stress. (b) Frequency spectrum of the specimen with residual stress.

The cross correlation coefficients of the IMFs of the specimen with and without the residual 
stress are calculated by Eq. (16). The results are shown in Tab.1.

Tab.1 The cross correlation coefficients of the IMFs

\begin{tabular}{cccccccc}
\hline$\rho_{1}$ & $\rho_{2}$ & $\rho_{3}$ & $\rho_{4}$ & $\rho_{5}$ & $\rho_{6}$ & $\rho_{7}$ & $\rho_{8}$ \\
\hline 0.5600 & 0.4897 & 0.4164 & 0.3524 & 0.1388 & 0.3623 & 0.3533 & 0.4179 \\
\hline
\end{tabular}

From Tab. 1 we can know that compared with other cross correlation coefficients, $\rho_{5}=0.1388$ is minimum and close to 0 . It indicates that in two different stress conditions, the correlation of the IMF5 is smaller, and the residual stress has great influence to the IMF5 component.

To calculate the local marginal spectrum and the main frequency of each IMF in local marginal spectrum is shown in Fig.3. It proves that the frequency variation of IMF5 is maximum.

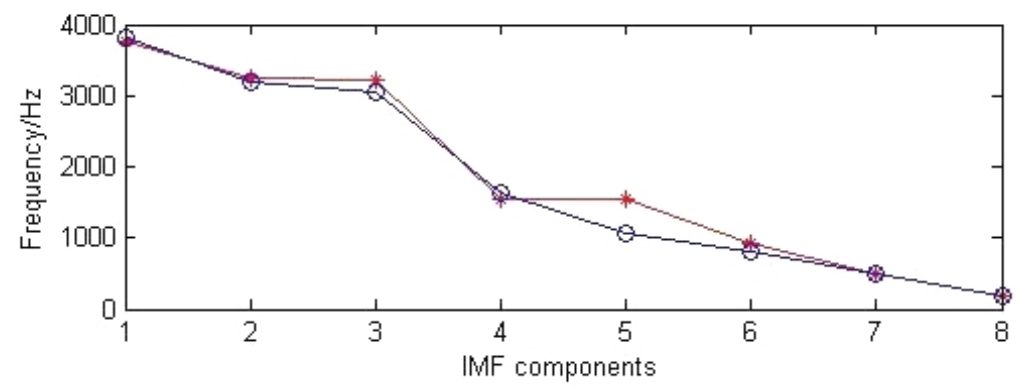

Fig.3 The main frequency of each IMF component in the local marginal spectrum

Due to the limitation of the size of the specimen and the stress level of the quenching, the residual stress has little effect on the low frequency of the sample. In many engineering applications, the working frequency of most parts is in the low frequency range, it will be difficult to attract the attention of researchers and operators. But for this method, we can know that the IMF5 component which is most affected by the residual stress can be obtained and it can provides a new way to judge the residual stress for this sample.

\section{Conclusions}

Using improved EMD method, the original vibration signals of the specimen with or without the residual stress can be decomposed into IMFs, and there are some relationship between the residual stress and the IMF components of the quenched aluminum alloy, the method proposed in this paper provides a new way to determine the existence of residual stress of this sample. It has great reference value and guiding significance to study the relationship between the residual stress and the IMF components of the sample.

\section{References}

[1] Huang X, Liu Z, Xie H. Recent progress in residual stress measurement techniques [J]. Acta Mechanica Solida Sinica. 2013, 26: 570-583.

[2] Rossini NS, Dassisti M, Benyounis KY, Olabi AG. Methods of measuring residual stresses in components [J]. Materials and Design. 2012, 35: 572-588.

[3] Seifi R, Salimi-Majd D. Effects of plasticity on residual stresses measurement by hole drilling method [J]. Mechanics of Materials. 2012, 53: 72-79.

[4] Zeinoddini M, Arnavaz S, Zandi AP, Alizadeh Vaghasloo Y. Repair welding influence on offshore pipelines residual stress fields: An experimental study [J]. Journal of Constructional 
Steel Research. 2013, 86: 31-41.

[5] Torres M E, Colominas M A, Schlotthauer G, et al. A complete ensemble empirical mode decomposition with adaptive noise [C] // IEEE International Conference on Speech and Signal Processing(ICASSP), May, 22-27, 2011, Prague, Czech. 2011: 4144-4147. 